\title{
Non-strictly black body spectrum from the tunnelling mechanism
}

\section{Christian Corda}

October 9, 2018

\author{
Institute for Theoretical Physics and Advanced Mathematics Einstein-Galilei, \\ Via Santa Gonda 14, 59100 Prato, Italy \\ E-mail address: cordac.galilei@gmail.com
}

\begin{abstract}
A modern and largely used approach to obtain Hawking radiation is the tunnelling mechanism. However, in various papers in the literature, the analysis concerned almost only to obtain the Hawking temperature through a comparison of the probability of emission of an outgoing particle with the Boltzmann factor.

In a interesting and well written paper, Banerjee and Majhi improved the approach, by explicitly finding a black body spectrum associated with black holes. On the other hand, this result, which has been obtained by using a reformulation of the tunnelling mechanism, is in contrast which the remarkable result by Parikh and Wilczek, that, indeed, found a probability of emission which is compatible with a non-strictly thermal spectrum.

By using our recent introduction of an effective state for a black hole, here we solve such a contradiction, through a slight modification of the analysis by Banerjee and Majhi. The final result will be a non-strictly black body spectrum from the tunnelling mechanism.

We also show that, for an effective temperature, we can write the corresponding effective metric by Hawking's periodicity arguments.

Potential important implications for the black hole information puzzle are also discussed.
\end{abstract}

In recent years, the tunnelling mechanism has been an elegant and largely used approach to obtain Hawking radiation [1, see for example [2- 6] and refs. within. Let us consider an object which is classically stable. If it becomes unstable from a quantum-mechanically point of view, one naturally suspects tunnelling. The mechanism of particles creation by black holes [1, can be described as tunnelling arising from vacuum fluctuations near the black hole's horizon [2]-6]. If a virtual particle pair is created just inside the horizon, the virtual particle with positive energy can tunnel out. Then, it materializes outside the black hole as a real particle. In the same way, if one considers a virtual 
particle pair created just outside the horizon, the particle with negative energy can tunnel inwards. In both of the situations, the particle with negative energy is absorbed by the black hole. The result will be that the mass of the black hole decreases and the particle with positive energy propagates towards infinity. Thus, subsequent emissions of quanta appear as Hawking radiation. A problem on such an approach was that, in the cited and in other papers in the literature, the analysis has been finalized almost only to obtain the Hawking temperature through a comparison of the probability of emission of an outgoing particle with the Boltzmann factor. The problem was apparently solved in the interesting work [7, where, through a reformulation of the tunnelling mechanism, a black body spectrum associated with black holes has been found. In any case, this result is in contrast which the remarkable result in [2, 3, that, indeed, found a probability of emission which is compatible with a non-strictly thermal spectrum. The non precisely thermal character of the spectrum has important implications for the black hole information puzzle as arguments that information is lost during black hole's evaporation partially rely on the assumption of strict thermal behavior of the spectrum [2, 3, 8, 9]. In fact, by introducing an effective state, we recently interpreted black hole's quasi-normal modes in terms of quantum levels by finding a natural connection between Hawking radiation and quasi-normal modes [8, 9]. As for large $n$ black holes result to be well defined quantum mechanical systems, having ordered, discrete quantum spectra, the results in [8, 9] look consistent with the unitarity of the underlying quantum gravity theory and with the idea that information should come out in black hole's evaporation.

Here we show that the effective quantities permit also to solve the above cited contradiction, through a slight modification of the analysis in [7]. The final result will be a non-strictly black body spectrum from the tunnelling mechanism.

For the sake of simplicity, in this letter we refer to the Schwarzschild black hole and we work with $G=c=k_{B}=\hbar=\frac{1}{4 \pi \epsilon_{0}}=1$ (Planck units).

Let us consider a Schwarzschild black hole. The Schwarzschild line element is (see [11] for clarifying historical notes to this notion)

$$
d s^{2}=-\left(1-\frac{2 M}{r}\right) d t^{2}+\frac{d r^{2}}{1-\frac{2 M}{r}}+r^{2}\left(\sin ^{2} \theta d \varphi^{2}+d \theta^{2}\right) .
$$

The event horizon is defined by $r_{H}=2 M$ [7, 10], while $\frac{1}{4 M}$ is the black hole's surface gravity. As we want to discuss Hawking radiation like tunnelling, the radial trajectory is relevant [2, 3, 7]. The analysis in [7] permitted to write down the (normalized) physical states of the system for bosons and fermions as [7]

$$
\begin{gathered}
\left|\Psi>_{\text {boson }}=(1-\exp (-8 \pi M \omega))^{\frac{1}{2}} \sum_{n} \exp (-4 \pi n M \omega)\right| n_{\text {out }}^{(L)}>\otimes \mid n_{\text {out }}^{(R)}> \\
\left|\Psi>_{\text {fermion }}=(1+\exp (-8 \pi M \omega))^{-\frac{1}{2}} \sum_{n} \exp (-4 \pi n M \omega)\right| n_{\text {out }}^{(L)}>\otimes \mid n_{\text {out }}^{(R)}>.
\end{gathered}
$$

Hereafter we focus the analysis only on bosons. In fact, for fermions the analysis is identical [7]. The density matrix operator of the system is [7] 


$$
\begin{gathered}
\hat{\rho}_{\text {boson }} \equiv \Psi>_{\text {boson }}<\left.\Psi\right|_{\text {boson }} \\
=(1-\exp (-8 \pi M \omega)) \sum_{n, m} \exp [-4 \pi(n+m) M \omega]\left|n_{\text {out }}^{(L)}>\otimes\right| n_{\text {out }}^{(R)}><m_{\text {out }}^{(R)}\left|\otimes<m_{\text {out }}^{(L)}\right| .
\end{gathered}
$$

If one traces out the ingoing modes, the density matrix for the outgoing (right) modes reads [7]

$$
\hat{\rho}_{\text {boson }}^{(R)}=(1-\exp (-8 \pi M \omega)) \sum_{n} \exp (-8 \pi n M \omega)\left|n_{\text {out }}^{(R)}><n_{\text {out }}^{(R)}\right| .
$$

This implies that the average number of particles detected at infinity is [7]

$$
<n>_{\text {boson }}=\operatorname{tr}\left[\hat{n} \hat{\rho}_{\text {boson }}^{(R)}\right]=\frac{1}{\exp (8 \pi M \omega)-1},
$$

where the trace has been taken over all the eigenstates and the final result has been obtained through a bit of algebra, see [7] for details. The result of eq. (5) is the well known Bose-Einstein distribution. A similar analysis works also for fermions [7, and one easily gets the well known Fermi-Dirac distribution

$$
<n>_{\text {fermion }}=\frac{1}{\exp (8 \pi M \omega)+1},
$$

Both the distributions correspond to a black body spectrum with the Hawking temperature [1, 7]

$$
T_{H} \equiv \frac{1}{8 \pi M} .
$$

The result in [7, that we shortly reviewed, is remarkable. In fact, through a reformulation of the tunnelling mechanism, one can found a black body spectrum associated with black holes which is in perfect agreement with the famous original result by Hawking [1]. On the other hand, it is in contrast with another remarkable result [2, 3]. In fact, the probability of emission connected with the two distributions (21) and (22) is given by [1, 2, 3]

$$
\Gamma \sim \exp \left(-\frac{\omega}{T_{H}}\right)
$$

But in [2, 3] a remarkable correction, through an exact calculation of the action for a tunnelling spherically symmetric particle, has been found, yielding

$$
\Gamma \sim \exp \left[-\frac{\omega}{T_{H}}\left(1-\frac{\omega}{2 M}\right)\right]
$$

This important result, which is clearly in contrast with the result in [7], enables a correction, the additional term $\frac{\omega}{2 M}$ [2, 3]. The important difference is that the authors of [7] did not taken into due account the conservation of the energy, which generates a dynamical instead of static geometry of the black hole [2, 3]. 
In other words, the energy conservation forces the black hole to contract during the process of radiation [2, 3]. Therefore, the horizon recedes from its original radius, and, at the end of the emission, the radius becomes smaller [2, 3]. The consequence is that black holes do not strictly emit like black bodies [2, 3].

It is important to recall that the tunnelling is a discrete instead of continuous process [8]. In fact, two different countable black hole's physical states must be considered, the physical state before the emission of the particle and the physical state after the emission of the particle [8]. Thus, the emission of the particle can be interpreted like a quantum transition of frequency $\omega$ between the two discrete states [8]. In the language of the tunnelling mechanism, a trajectory in imaginary or complex time joins two separated classical turning points [2, 3]. Another important consequence is that the radiation spectrum is also discrete [8]. Let us clarify this important issue in a better way. At a well fixed Hawking temperature and the statistical probability distribution (9) are continuous functions. On the other hand, the Hawking temperature in (9) varies in time with a character which is discrete. In fact, the forbidden region traversed by the emitting particle has a finite size [3]. Considering a strictly thermal approximation, the turning points have zero separation. Therefore, it is not clear what joining trajectory has to be considered because there is not barrier [3. The problem is solved if we argue that the forbidden finite region from $r_{\text {initial }}=2 M$ to $r_{\text {final }}=2(M-\omega)$ that the tunnelling particle traverses works like barrier [3]. Thus, the intriguing explanation is that it is the particle itself which generates a tunnel through the horizon 3 .

A good way to take into due account the dynamical geometry of the black hole during the emission of the particle is to introduce the black hole's effective state. By introducing the effective temperature [8, 9]

$$
T_{E}(\omega) \equiv \frac{2 M}{2 M-\omega} T_{H}=\frac{1}{4 \pi(2 M-\omega)},
$$

one re-writes eq. (10) in a Boltzmann-like form similar to the original probability found by Hawking

$$
\Gamma \sim \exp \left[-\beta_{E}(\omega) \omega\right]=\exp \left(-\frac{\omega}{T_{E}(\omega)}\right),
$$

where $\exp \left[-\beta_{E}(\omega) \omega\right]$ is the effective Boltzmann factor, with [8, 9]

$$
\beta_{E}(\omega) \equiv \frac{1}{T_{E}(\omega)} .
$$

Hence, the effective temperature replaces the Hawking temperature in the equation of the probability of emission [8, 9]. Let us discuss the physical interpretation. In various fields of science, we can takes into account the deviation from the thermal spectrum of an emitting body by introducing an effective temperature. It represents the temperature of a black body that would emit the same total amount of radiation. We introduced the concept of effective temperature in the black hole's physics in [8, 9. $T_{E}(\omega)$ depends on the energy-frequency of 
the emitted radiation and the ratio $\frac{T_{E}(\omega)}{T_{H}}=\frac{2 M}{2 M-\omega}$ represents the deviation of the radiation spectrum of a black hole from the strictly thermal feature [8, 9].

The introduction of $T_{E}(\omega)$ permits the introduction of others effective quantities. In fact, let us consider the initial mass of the black hole before the emission, $M$, and the final mass of the hole after the emission, $M-\omega$ respectively [8, 9]. The effective mass and the effective horizon of the black hole during its contraction, i.e. during the emission of the particle, are defined as [8, 9]

$$
M_{E} \equiv M-\frac{\omega}{2}, r_{E} \equiv 2 M_{E}
$$

The above effective quantities are average quantities [8, 9. $r_{E}$ is the average of the initial and final horizons and $M_{E}$ is the average of the initial and final masses [8, 9]. Therefore, $T_{E}$ is the inverse of the average value of the inverses of the initial and final Hawking temperatures (before the emission $T_{H}$ initial $=\frac{1}{8 \pi M}$, after the emission $T_{H}$ final $=\frac{1}{8 \pi(M-\omega)}$ respectively) [8, 9]. Thus, the Hawking temperature has a discrete character in time.

We stress that the introduction of the effective temperature does not degrade the importance of the Hawking temperature. Indeed, as the Hawking temperature changes with a discrete behavior in time, it is not clear which value of such a temperature has to be associated to the emission of the particle. Has one to consider the value of the Hawking temperature before the emission or the value of the Hawking temperature after the emission? The answer is that one must consider an intermediate value, the effective temperature, which is the inverse of the average value of the inverses of the initial and final Hawking temperatures. In a certain sense, it represents the value of the Hawking temperature during the emission. $T_{E}(\omega)$ takes into account the non-strictly thermal character of the radiation spectrum and the non-strictly continuous character of subsequent emissions of Hawking quanta.

Therefore, one can define two further effective quantities. The effective Schwarzschild line element is given by

$$
d s_{E}^{2} \equiv-\left(1-\frac{2 M_{E}}{r}\right) d t^{2}+\frac{d r^{2}}{1-\frac{2 M_{E}}{r}}+r^{2}\left(\sin ^{2} \theta d \varphi^{2}+d \theta^{2}\right),
$$

and, consequently, the effective surface gravity is defined as $\frac{1}{4 M_{E}}$. Thus, the effective line element (14) takes into account the dynamical geometry of the black hole during the emission of the particle. Clearly, if one follows step by step the analysis in [7, at the end obtains the correct physical states for boson and fermions as

$$
\begin{gathered}
\left|\Psi>_{\text {boson }}=\left(1-\exp \left(-8 \pi M_{E} \omega\right)\right)^{\frac{1}{2}} \sum_{n} \exp \left(-4 \pi n M_{E} \omega\right)\right| n_{\text {out }}^{(L)}>\otimes \mid n_{\text {out }}^{(R)}> \\
\left|\Psi>_{\text {fermion }}=\left(1+\exp \left(-8 \pi M_{E} \omega\right)\right)^{-\frac{1}{2}} \sum_{n} \exp \left(-4 \pi n M_{E} \omega\right)\right| n_{\text {out }}^{(L)}>\otimes \mid n_{\text {out }}^{(R)}>
\end{gathered}
$$


and the correct distributions as

$$
\begin{gathered}
<n>_{\text {boson }}=\frac{1}{\exp \left(8 \pi M_{E} \omega\right)-1}=\frac{1}{\exp [4 \pi(2 M-\omega) \omega]-1} \\
<n>_{\text {fermion }}=\frac{1}{\exp \left(8 \pi M_{E} \omega\right)+1}=\frac{1}{\exp [4 \pi(2 M-\omega) \omega]+1}
\end{gathered}
$$

which represent the distributions associated to the probability of emission (11).

Again, we emphasize that this deviation from strict thermality is consistent with unitarity [3, 8, 9] and has profound implications for the black hole information puzzle because arguments that information is lost during black hole's evaporation rely in part on the assumption of strict thermal behavior of the spectrum [3, 8, 9, 17. In other words, the process of black hole's evaporation should be unitary, information should be preserved and the underlying quantum gravity theory should be unitary too.

The main issue in this letter is the logic behind writing the expression for the effective metric (14) [18. We show that for an effective temperature we can write the corresponding metric by Hawking's periodicity argument [14, 18, 19, 20]. Let us rewrite eq. (12) as

$$
\beta_{E}(\omega) \equiv \frac{1}{T_{E}(\omega)}=\beta_{H}\left(1-\frac{\omega}{2 M}\right),
$$

where $\beta_{H} \equiv \frac{1}{T_{H}}$. Following Hawking's arguments [19, 20] the euclidean form of the metric will be given by

$$
d s_{E}^{2}=x^{2}\left[\frac{d \tau}{4 M\left(1-\frac{\omega}{2 M}\right)}\right]^{2}+\left(\frac{r}{r_{E}}\right)^{2} d x^{2}+r^{2}\left(\sin ^{2} \theta d \varphi^{2}+d \theta^{2}\right),
$$

which is regular at $x=0$ and $r=r_{E} . \tau$ is treated as an angular variable with period $\beta_{E}(\omega)$ [19, 20]. Replacing the quantity $\sum_{i} \beta_{i} \frac{\hbar^{i}}{M^{2 i}}$ in [19] with the quantity $-\frac{\omega}{2 M}$, if one follows step by step the detailed analysis in [19] the modified eq. (14) is easily obtained and one also easily shows that $r_{E}$ in eq. (18) is the same as in eq. (13). Thus, Hawking's periodicity argument gives completeness to our analysis and will further endorse the correctness of the results.

Conclusion remarks In the remarkable paper [7] the tunnelling approach on Hawking radiation has been improved by explicitly finding a black body spectrum associated with black holes. But a problem is that this result, which has been obtained by using a reformulation of the tunnelling mechanism, is in contrast which the other remarkable result in [2, 3, that, indeed, found a probability of emission which is compatible with a non-strictly thermal spectrum.

By using our recent introduction of an effective state for a black hole [8, 9] in this paper we solved such a contradiction, through a slight modification of the analysis in [7. The final result consists in a non-strictly black body spectrum from tunnelling mechanism. 
We have also shown that, for an effective temperature, we can write the corresponding effective metric by Hawking's periodicity arguments. This point gave completeness to our analysis and further endorsed the correctness of the results.

Potential important implications for the black hole information puzzle have been also discussed. In fact, arguments that information is lost during black hole's evaporation partially rely on the assumption of strict thermal behavior of the spectrum [2, 3, 8, 9. Hence, this letter completes our previous results in [8, 9] which, by showing the black hole in terms of a well defined quantum mechanical system, having an ordered, discrete quantum spectrum, look consistent with the unitarity of the underlying quantum gravity theory and with the idea that information should come out in black hole's evaporation.

Acknowledgements The author thanks an anonymous referee for useful criticisms and comments which permitted to improve this letter. The author also

thanks Doug Singleton and Sujoy Modak for signalling some typos that have been correct in this final version of the letter.

\section{References}

[1] S. W. Hawking, Commun. Math. Phys. 43, 199 (1975).

[2] M. K. Parikh and F. Wilczek, Phys. Rev. Lett. 85, 5042 (2000).

[3] M. K. Parikh, Gen. Rel. Grav. 36, 2419 (2004, First Award in the Gravity Research Foundation Competition).

[4] R. Banerjee and B.R. Majhi, JHEP 0806, 095 (2008).

[5] M. Angheben, M. Nadalini, L. Vanzo and S. Zerbini, JHEP 0505, 014 (2005).

[6] M. Arzano, A. J. M. Medved and E. C. Vagenas, JHEP 0509, 037 (2005).

[7] R. Banerjee and B.R. Majhi, Phys. Lett. B 675, 243 (2009).

[8] C. Corda, Int. Journ. Mod. Phys. D 21, 1242023 (2012, Honorable Mention in the Gravity Research Foundation Competition).

[9] C. Corda, JHEP 1108, 101 (2011).

[10] C. W. Misner, K. S. Thorne, J. A. Wheeler, "Gravitation", Feeman and Company (1973).

[11] C. Corda, Electr. J. Theor. Phys. 8, 25, 65-82 (2011).

[12] K. Srinivasan and T. Padmanabhan, Phys. Rev. D 60, 024007 (1999).

[13] B. R. Majhi, Phys. Rev. D 79, 044005 (2009). 
[14] B. R. Majhi and S. Samanta, Annals Phys. 325, 2410 (2010).

[15] R. Banerjee and B. R. Majhi, Phys. Rev. D 79, 064024 (2009).

[16] V. Akhmedova, T. Pilling, A. Gill and D. Singleton, Phys. Lett. B 666, 269 (2008).

[17] S. W. Hawking, Phys. Rev. D72, 084013 (2005).

[18] Private communication with the referee.

[19] R. Banerjee and B. R. Majhi, Phys. Lett. B 674, 218 (2009).

[20] S.W.Hawking, "The Path Integral Approach to Quantum Gravity", in General Relativity: An Einstein Centenary Survey, eds. S.W.Hawking and W.Israel, (Cambridge University Press, 1979). 